\title{
Humo de Tabaco Ambiental y Neumonías en Niños de Monterrey, México
}

\section{Environmental tobacco smoke and pneumonia in children living in Monterrey, México}

Sixto F. Gutiérrez-Ramírez ${ }^{1}$, Gloria M. Molina-Salinas², Jaime F. García-Guerra ${ }^{1}$, Javier Vargas-Villarreal², Benito D. Mata-Cárdenas² y Francisco González-Salazar²

1. Hospital Metropolitano "Dr. Bernardo Sepúlveda", Nuevo León, México.

drsixtogrped@hotmail.com,drsixtogrped@hotmail.com

2. Centro de Investigación Biomédica del Noreste, Instituto Mexicano del Seguro Social.

Monterrey, Nuevo León, México. gmolina70@hotmail.com,jvargas147@yahoo.com.mx, bdmata@hotmail.com,fgonz75@yahoo.com.mx

Recibido 13 Abril 2006/Enviado para Modificación 11 Noviembre 2006/Aprobado 23 Enero 2007

\section{RESUMEN}

Objetivo Las enfermedades respiratorias agudas ocupan en el mundo los primeros 5 lugares en morbilidad y mortalidad infantil, anualmente mueren más de dos millones de niños por esa causa. El humo del tabaco ambiental (HTA) contiene compuestos irritantes y tóxicos nocivos para la salud, que incrementan el riesgo de morbilidad y mortalidad en lactantes, niños y adultos no fumadores. Nuestro principal objetivo fue determinar la asociación entre HTA y la presencia de neumonías en niños.

Material y métodos Se realizó un estudio de casos y controles no pareados. Se calcularon las razones de momios (RM) e intervalo de confianza $95 \%$ (IC) para cada variable.

Resultados Se analizaron 285 pacientes, 142 casos y 143 controles, 47,4\% femeninos, la edad promedio fue 4,5+2,7 años. La RM para expuestos al HTA fue 3,44, IC 2,1- 5,6.

Discusión La exposición a HTA incrementa más de tres veces el riesgo de padecer neumonías en niños.

Palabras Clave: Infecciones del tracto respiratorio, neumonías, niños, humo de tabaco, contaminación del aire por humo de tabaco (fuente: DeCS, BIREME).

\section{ABSTRACT}

Objective Acute respiratory diseases occupy the first 5 places in infantile morbidity and mortality around the world, two million children directly dying from such cause annually. Environmental tobacco smoke (ETS) contains toxic and irritating compounds having an injurious effect on health, producing increased risk of morbidity and mortality in non-smoking adults and children. Our main objective was 
determining the association between ETS and pneumonia in children. Material and methods This was an unmatched case and controls hospital-based study. Odds ratio $(\mathrm{OR})$ and $95 \%$ confidence interval $(\mathrm{Cl})$ were calculated.

Results A total of 285 patients (142 cases and 143 controls) were studied; $47,4 \%$ of the patients were female and average age was $4,5,+2,7$. OR for patients being exposed to ETS developing pneumonia was 3,44 (Cl: 2,11-5,6).

Discussion Children being exposed to ETS increases the risk of developing pneumonia by more than threefold.

Key Words: Respiratory tract infection, pneumonia, children, environmental tobacco smoke, air pollution (source: $M e S H, N L M$ ).

L as enfermedades respiratorias agudas ocupan los primeros 5 lugares en morbilidad y mortalidad infantil a nivel mundial, además según la OMS se reconoce que anualmente mueren en el mundo cerca de 2100000 niños con diagnóstico de infecciones respiratorias agudas, el $85 \%$, de ellas son neumonías (1). En México ocurren cerca de 200000 casos de neumonía por año; esta es la segunda causa de muerte en niños lactantes y preescolares y la cuarta causa de muerte en escolares (2). El humo del tabaco presente en el ambiente (HTA) también conocido como humo de segunda mano es una mezcla compleja de más de 4000 compuestos que son generados durante el quemado de los productos del tabaco como cigarro, puro y pipa. Esta mezcla contiene numerosos irritantes y tóxicos con efectos agudos para la salud así como tóxicos con efectos carcinogénicos para humanos (3). Es conocido el incremento del riesgo de morbilidad y mortalidad en lactantes, niños y adultos no fumadores expuestos a HTA (4). El HTA juega un papel importante en la salud de los niños, así tenemos que uso de productos del tabaco produce un impacto enorme sobre ellos, pues existe asociación con enfermedades en vías respiratorias altas y bajas, lesiones relacionadas a fuego, episodios de otitis media, timapnostomías, amigdalectomías y adenoidectomías, asma, tos, bronquitis, pulmonía incluso muerte en niños (5). El efecto del HTA parece cambiar dependiendo de la edad de los niños estudiados; la mayoría de los estudios han sido enfocados en el primer año de vida (6-8).

Existen reportes de una alta exposición en la población estadounidense donde el $43 \%$ de niños con edades entre dos meses y 11 años vivieron en un hogar con al menos un fumador y el $37 \%$ de los adultos no fumadores vivieron con un fumador o reportaron exposición al HTA (9). Recientemente ha sido reportado que entre el 20 y $40 \%$ de los hogares estadounidenses 
contribuyen a la exposición de HTA para las personas no fumadoras (10). La ruta mejor conocida de exposición al HTA es la inhalación de aire contaminado (11).

La inhalación de HTA mientras un cigarro está siendo fumado es la forma de exposición principal y pero no la única. Así, tenemos que los componentes del HTA son rápidamente dispersos como partículas tóxicas con un diámetro menor a 2,5 micrómetros después de su emisión y están disponibles para reaccionar químicamente. Estas partículas, pueden ser respirables y no removibles por los mecanismos protectores de las mucosas respiratorias (1214).

El polvo presente en hogar y las superficies contaminadas son las mayores fuentes de contaminantes como plomo, alergenos, pesticidas e hidrocarburos aromáticos policíclicos (15). Hein y colaboradores (16) fueron los primeros en publicar la presencia de nicotina en el polvo respirable de hogares de personas fumadoras. Los componentes de HTA en fase de vapor son depositados y adsorbidos en paredes, muebles del hogar, ropa, juguetes y otros objetos dentro de los 10 minutos hasta horas después que el humo de tabaco ha sido emitido. Además, éstos pueden ser re-emitidos al ambiente en horas hasta meses después del consumo, por lo cual se dice que: es posible una re-contaminación ambiental dinámica entre el polvo de tapetes, paredes, muebles y otros objetos del hogar por semanas hasta varios meses posteriores a que los contaminantes fueron emitidos del cigarro $(12,13,17)$. Los niños hijos de padres fumadores están en particular riesgo de exposición al HTA a través del polvo contaminado presente en su casa. Se estima que la incorporación de polvo por los infantes es de 0,052,5 g/día, dos veces mas que el valor que para los adultos (15).

Los niños expuestos al HTA tienen mayor propensión a padecer tos nocturna, flema excesiva, silbidos en el pecho y mayor mucosidad (18). Gryczynska D (19) reporta que los niños expuestos al HTA padecen más infecciones de vías respiratorias altas que los niños no expuestos. Además, los niños expuestos al HTA son más propensos a desarrollar asma o de mayor severidad o dificultad para controlarla (20).

Este estudio, explora el papel de la exposición de niños a los componentes del HTA y desarrollo de neumonías, pues no existen en nuestro medio estudios relacionados con este tema. 


\section{MATERIAL Y MÉTODOS}

Se realizó un estudio de casos y controles en el Hospital Metropolitano Dr. Bernardo Sepúlveda, ubicado en la zona norte del área metropolitana de Monterrey, Nuevo León, México. Este es un hospital de concentración de segundo nivel de los Servicios de Salud de la Secretaría de Salud de Nuevo León. El estudio se realizó durante el período de Marzo de 2004 a Febrero de 2005 en las áreas de hospitalización, urgencias y consulta externa del Servicio de Pediatría del mencionado hospital. La población que acude a nuestro hospital es referida de los Centros de Salud de zona urbana y rural de nuestro estado; generalmente no cuentan con seguridad social y pertenecen al nivel socioeconómico bajo o medio bajo. Nuestra muestra representa a la población sin asistencia social de nuestro estado. Calculamos un tamaño de muestra de 285 pacientes con la ayuda del programa STATS v 1.1 para obtener un nivel de confianza de $95 \%$, potencia de prueba de $80 \%$ y permitir identificar una diferencia del grado de exposición entre los casos y los controles de un $30 \%$. Consideramos formar 2 grupos; el primer grupo al cual denominamos casos, fue formado por 142 pacientes niños menores de 10 años hospitalizados con diagnóstico de neumonía, el segundo grupo denominado controles en el cual se incluyeron 143 pacientes menores de 10 años de edad seleccionados al azar de los pacientes sanos que acudieron a la consulta externa. A los padres de los pacientes de ambos grupos se les invitó a participar en el estudio y posterior a la firma del consentimiento informado se colectó la información pertinente. Para cada caso de un niño con diagnóstico de neumonía se seleccionó un control sin neumonía, sin considerar edad ni sexo de los pacientes. El diagnóstico de neumonía (caso) se estableció clínica y radiológicamente. Los hallazgos clínicos que se consideraron para sospechar neumonía fueron: fiebre, ataque al estado general, tos húmeda, presencia de estertores crepitantes en la auscultación con o sin dificultad respiratoria y la confirmación del diagnóstico se realizó por radiografía de tórax postero-anterior donde se mostraron infiltrados pulmonares alveolares o nodulares. Este diagnóstico se confirmó por consenso de los pediatras del área, un medico externo y dos o mas residentes de pediatría. Los controles fueron pacientes clínicamente sanos.

A todos los pacientes se les evaluó además el grado de exposición al humo de tabaco ambiental (HTA). Interrogando de manera directa a los padres acerca del consumo de cigarrillos, puros, pipas o cualquier forma de consumo de tabaco inhalado por personas mayores o menores de edad, en los últimos seis meses dentro o fuera de la vivienda donde regularmente duerme el menor incluido 
en el estudio. Se considero expuesto a todo aquel paciente que los padres referían cohabitaba con personas mayores o menores de edad consumidores de tabaco inhalado en los últimos seis meses dentro o fuera de la vivienda donde regularmente dormía el menor incluido en el estudio.

Se analizaron además otras variables: Las variables independientes edad, edad materna, sexo, internamiento previo y asistencia ventilatoria, las cuales se definieron como sigue: la edad se registró en años de acuerdo al número de años cumplidos del niño al momento de realizar la entrevista; la edad materna se registró en años de acuerdo a los años que haya cumplido la madre del paciente al momento de realizar el estudio; el sexo se registró como masculino o femenino de acuerdo a como lo mencionó la madre del menor al momento de realizar la entrevista; se interrogó sobre el antecedente de internamiento por neumonía del menor investigado y fue utilizada escala nominal, con los valores si o no. La variable internamiento previo también se consideró como variable dependiente para asociar en tablas cruzadas con exposición HTA. Para la variable asistencia ventilatoria se consideró que un niño la requirió cuando se utilizaron puntas nasales, mascarilla, casco o intubación durante su estancia hospitalaria actual; esta se registró como variable nominal y los valores fueron si o no.

Criterios de selección:

Criterios de exclusión. Niños mayores de 10 años, niños fumadores, pacientes con asma, cardiopatías congénitas o adquiridas, niños con broncodisplasia, mucovisidosis, hijos de madres alcohólicas o drogadictas y pacientes con malformaciones de tórax.

Criterios de eliminación. Se eliminaron del estudio aquellos pacientes que no acudieron a las citas para colectar las muestras de sangre y aquellos que por cualquier motivo no aceptaron vacunarse.

Análisis estadístico de los datos. Los datos obtenidos se analizaron usando el software SPSS versión 10.0. Los promedios se compararon usando la $t$ de student, y las frecuencias de las variables cualitativas, usando Chi cuadrado; la fuerza de asociación se evaluó usando la razón de momios (RM), para cada RM se calcularon los intervalos de confianza de 95\%, consideramos significativo un valor de p menor a 0.05 . 


\section{RESULTADOS}

Se evaluaron 285 pacientes, 142 casos y 143 controles, 52,6 \% del total de pacientes fueron hombres y el 47,4 \% fueron mujeres. Cuando se compararon los porcentajes del género de ambos grupos no se encontraron diferencias significativas entre ellos, $p=0.20$. La edad promedio del total de pacientes fue de 4,56 $\pm 2,72$. La edad promedio de los casos fue de 4,37 $\pm 2,56$ mientras que la de los controles fue de 4,74 $\pm 2,88$. Al comparar las medias de las edades tampoco encontramos diferencias significativas $\mathrm{p}=0.25$. La edad promedio de las madres en la población analizada fue de 27,8 $\pm 4,9$ las madres de los casos tenían un promedio de edad de 28,01 \pm 4.8 mientras que la de los controles fue de $27,77 \pm 5,1$, no se encontraron diferencias significativas entre la edad de las madres de los casos y los controles $\mathrm{p}=0.68$. La comparación de los promedios de los grupos de las variables cuantitativas puede verse en la Tabla 1 . El porcentaje de exposición a HTA en la población estudiada fue de 52,4 \%, contrastando con $67,6 \%$ en los casos y de 37,8 \% en los controles, la significancia estadística al comparar los grupos fue $\mathrm{p}<0.001$. El 17,5\% de los pacientes tenían antecedentes de haber estado internados por neumonía, el 23,2 \% de los casos tenían una historia de haber estado internados por el mismo diagnóstico y sólo el 11,9 \% de los controles tenían ese mismo antecedente (Tabla 2). Cuando consideramos la exposición a HTA el 24,7 \% de los pacientes tenían antecedentes de haber sido internados por neumonía mientras los no expuestos solo el 9,6 \%. El 13,4 $\%$ de los casos requirieron asistencia ventilatoria, de ellos el $79 \%$ estaba expuesto a HTA mientras el $21 \%$ no lo estaba, $\mathrm{p}<0.001$.

Las RM para analizar la asociación entre las variables independientes y el desarrollo de neumonía que requiere internamiento se muestra en la Tabla 3. Ahí podemos observar como la edad, el sexo, la edad de la madre, no son factores que muestren diferencias significativas al comparar el grupo de casos con los controles. Mientras que la historia de internamientos previos por neumonía y exposición a HTA mostró una RM de 2,77 y 3,28 respectivamente, la significancia estadística fue de 0.005 y 0.001 . Cuando se realizaron tablas cruzadas usando la variable asistencia ventilatoria como variable dependiente, la RM que mostraron los expuestos a HTA fue de 3,63 (IC 95\% 1,17-11,25) con un valor de $\mathrm{p}=0.02$, mientras que cuando se realizaron tablas cruzadas usando como variable dependiente la variable "internamiento previo por neumonía", la razón de momios para los casos expuestos a HTA fue de 3,07 (IC 95\% 1,55-6,07) con un valor de $\mathrm{p}<0.001$. 
REVISTA DE SALUD PÚBLICA · Volumen 9(1), Marzo 2007

Tabla 1. Comparación de medias de variables cuantitativas

\begin{tabular}{lccc}
\hline \multicolumn{1}{c}{ Variable } & $\begin{array}{c}\text { Razón de } \\
\text { momios }\end{array}$ & IC 95\% & Significancia \\
\hline Edad & 0,904 & $1,01-0,809$ & 0,074 \\
Sexo & 0,992 & $1,63-0,603$ & 0,976 \\
Edad materna & 1,047 & $1,11-0,986$ & 0,136 \\
Expuesto a HTA & 3,280 & $5,41-1,98$ & 0,001 \\
Internamiento previo & 2,778 & $5,69-1,35$ & 0,005 \\
\hline
\end{tabular}

Tabla 2. Comparación de frecuencias de variables cualitativas

\begin{tabular}{lccccc}
\hline Variable & Parámetro & Casos & Controles & Población & $\begin{array}{c}\text { Significancia } \\
\mathrm{p}=\end{array}$ \\
\hline \multirow{2}{*}{ Edad } & Promedio & 4,37 & 4,74 & 4,56 & 0,25 \\
& Desviación estándar & 2,56 & 2,88 & 2,72 & \\
\hline Edad & Promedio & 28,01 & 27,77 & 27,89 & 0,68 \\
Materna & Desviación estándar & 4,86 & 5,12 & 4,98 & \\
\hline
\end{tabular}

Tabla 3. Razones de momios para el riesgo de desarrollar Neumonía en niños

\begin{tabular}{|c|c|c|c|c|c|}
\hline Variable & Indicador & $\begin{array}{c}\text { Casos } \\
(\%)\end{array}$ & $\begin{array}{c}\text { Controles } \\
(\%)\end{array}$ & $\begin{array}{c}\text { Población } \\
(\%)\end{array}$ & $\begin{array}{c}\text { Significancia* } \\
p=\end{array}$ \\
\hline \multirow{2}{*}{ Género } & Masculino & 51,4 & 53,8 & 52,6 & \multirow{2}{*}{0,68} \\
\hline & Femenino & 48,6 & 46,2 & 47,4 & \\
\hline \multirow{2}{*}{$\begin{array}{l}\text { Exposición a } \\
\text { humo de } \\
\text { abaco }\end{array}$} & $\mathrm{Si}$ & 67,6 & 37,8 & 52,4 & \multirow{2}{*}{0,001} \\
\hline & No & 32,4 & 62,2 & 47,6 & \\
\hline \multirow{2}{*}{$\begin{array}{l}\text { Internamiento } \\
\text { previo }\end{array}$} & $\mathrm{Si}$ & 23,2 & 11,9 & 17,5 & \multirow{2}{*}{0,011} \\
\hline & No & 76,8 & 88,1 & 82,5 & \\
\hline \multirow{2}{*}{$\begin{array}{l}\text { Asistencia } \\
\text { ventilatoria }\end{array}$} & $\mathrm{Si}$ & 13,4 & 0 & 6,7 & \multirow{2}{*}{0,001} \\
\hline & No & 86,6 & 100 & 97,3 & \\
\hline
\end{tabular}

\section{DISCUSIÓN}

A pesar de que se conocen los daños que causa el cigarro a las personas fumadoras y a los fumadores pasivos (18-20), la frecuencia de exposición al HTA a nuestros pacientes fue de 52,6 \% para la población estudiada y de hasta $67.6 \%$ en el grupo de casos es una frecuencia bastante mayor a la reportada en Estados Unidos y otros países europeos donde la frecuencia se estima entre un 20 y 40 \% (10). En nuestro país no se había reportado nunca acerca de la frecuencia de exposición al HTA en niños con neumonías. Nosotros 
encontramos una asociación de 3,4 veces más neumonías en pacientes expuestos. Además encontramos que los pacientes internados por neumonía tienen un riesgo mayor de tres veces de volver a requerir internarse nuevamente por este diagnóstico, es decir que los niños menores de 10 años y expuestos a humo de tabaco tienen un riesgo superior a tres veces de estar internados por lo menos dos veces en ese periodo de su infancia. Luego, los niños internados con diagnóstico de neumonía y expuestos a HTA tienen 3,6 veces más posibilidades de requerir algún tipo de soporte ventilatorio comparados con los niños no expuestos. Si consideramos que las infecciones respiratorias agudas son de las primeras 5 causas de consulta y de muerte en los niños y que las neumonías producen en el mundo más de 1850000 muertes por año y más del $87 \%$ ocurren en países en desarrollo (1), entonces podemos inferir que como en nuestro estudio encontramos asociación de exposición a HTA en más del $67 \%$ de los casos y de las personas expuestas por lo menos el $64 \%$ de ellos pueden desarrollar neumonía en los primeros 10 años de vida. El reducir la exposición al HTA en los hogares es una medida preventiva a establecer de manera urgente y definitiva. Con ello buscamos disminuir la incidencia de neumonías en niños y la mortalidad por esta causa. Se requiere una mayor cantidad de estudios que nos permitan establecer los mecanismos por los cuales se favorece la mayor predisposición de los pacientes expuestos a padecer neumonía.

Hay que considerar que nuestro hospital es un hospital de referencia al cual acuden hijos de padres sin asistencia social, es decir sin un trabajo asalariado permanente, sin embargo los hábitos de consumo de tabaco pueden no estar afectados por esa condición. No así, otros factores no evaluados en este estudio como el nivel de educación, nivel socioeconómico y condiciones de vivienda, los cuales podrían ser evaluados en estudios posteriores.

No se contempló la intensidad de exposición, el tiempo de exposición, ni la cantidad de personas fumadoras que cohabitan con el menor enfermo. Sin embargo, si se pudo establecer que la exposición de un menor con por lo menos una persona mayor o menor de edad consumidor de tabaco inhalado en los últimos seis meses dentro o fuera de la vivienda donde regularmente duerme incrementa más de tres veces su riesgo de desarrollar neumonías.

Aunque las neumonías no dependen únicamente de la exposición al humo del tabaco, sino de otros factores no evaluados en el presente estudio, hay que considerar el HTA como un factor de riesgo para desarrollar esta enfermedad. 
Se insiste en la necesidad de invitar a los padres de los menores a dejar de fumar, y aunque poco más del $40 \%$ de los fumadores tienen dependencia a la nicotina (21), por lo menos deberán de dejar de fumar dentro o fuera de su casa y/o en lugares donde convivan con sus hijos como por ejemplo en el automóvil.

Podemos concluir que la exposición a HTA en niños menores de 10 años incrementa más de tres veces el riesgo de padecer neumonías. Además, el haber padecido una neumonía y tener antecedente de exposición a HTA incrementa más de dos veces el riesgo de padecer nuevamente neumonía. En los pacientes con neumonía, la exposición a HTA incrementa mas de tres veces el riesgo de requerir apoyo ventilatorio durante el internamiento. Por último; los pacientes expuestos a HTA tienen un riesgo dos veces superior a los no expuestos de presentar por lo menos 2 internamientos por neumonía en sus primeros 10 años de vida

\section{REFERENCIAS}

1. World Health Organization [Internet]. Global Health Report 2005. Disponible en: http:/ /www.who.int/whr/2005. Consultado noviembre 2006.

2. Panamerican Health Organization [Internet]. America's Global Health 2004. Disponible en: http://www.paho.org/spanish/sha/prflmex.htm. Consultado noviembre 2006.

3. Matt GE Quintana PJ, Hovell MF, Bernert JT, Song S, Novianti N, et al. Households contaminated by environmental tobacco smoke: sources of infant exposures. Tob Control 2004 ;13(1):29-37.

4. US Environmental Protection Agency. Environmental tobacco smoke. Health effects and prevention policies. Council on Scientific Affairs, American Medical Association. Arch Fam Med. 1994; 3(10):865-71.

5. DiFranza JR, Lew RA. Morbility and mortality in children associated with the use of tobacco products by other people. Pediatrics 1996; 97 (4): 560-8.

6. Harlap S, Davies AM. Infant admissions to hospital and maternal smoking. Lancet 1974;1(7857): 529-532.

7. Pedreira FA, Guandolo VL, Feroli EJ, Mella GW, Weiss IP. Involuntary smoking and incidence of respiratory illness during the first year of life. Pediatrics 1985; 75(3): 5947

8. Nafstad P, Jaakkola JJK, Hagen JA, Botten G, Kongerud J. Breastfeeding, maternal smoking and lower respiratory tract infections. Eur Respir J 1996;9(12): 2623-9.

9. Prinkle JL, Flegal KM, Bernet JT. Exposure of the US population to environmental tobacco smoke: The Third Nacional Health and Nutrition Examination Survey, 1998 to 1991. JAMA 1996; 275 (16): 1233-40. 
10. US Centers for Disease Control and Prevention. State-specific prevalence of current cigarette smoking among adults and policies and attitudes about secondhand smoke-United States, 2000. JAMA 2002; 287(3):309-10.

11. California Environmental Protection Agency. Health effects of exposure to environmental tobacco smoke. California Environmental Protection Agency. Tob Control. 1997; 6(4):346-53.

12. Daisey JM Tracers for assessing exposure to environmental tobacco smoke: what are they training? Environ Health Perspect 1999;107 Suppl 2:319-27.

13. Daisey JM, Angell WJ, Apte MG. Indoor air quality, ventilation and health symptoms in schools: an analysis of existing information. Indoor Air 2003;13(1):53-64.

14. Cohen D, Arai SF, Brain JD. Smoking impairs long-term dust clearance form the lung. Science 1979; 204(4392):514-7.

15. Roberts JW, Dickey P. Exposure of children to pollutants in house dust and indoor air. Rev Environ Contam Toxicol 1995; 143: 59-78.

16. Hein HO, Suadicani P, Skov P et al. Indoor dust exposure: an unnoticed aspect of involuntary smoking. Arch Environ Health. 1991; 46 (2):98-101.

17. Van Loy MD, RileyWJ, Daisey JM, Nazaroff WW. Dynamic behavior of semivolatile organic compounds in indoor air. 2. Nicotine and phenanthrene with carpet and wallboard. Environ Sci Technol 2001;35(3): 560-7.

18. Cook DG, Strachan DP. Summary of effects of parental smoking on respiratory health of children and implications for research. Thorax 1999;54 (5):352-7.

19. Gryczynska D, Kobos J, Zakrzewska A. Relationship between passive smoking, recurrent respiratory tract infections and otitis media in children. Int J Pediatr Otorhinolaryngol 1999;5 Suppl 1:275-8.

20. Gilliland FD, Li YF, Peters JM. Effects of maternal smoking during pregnancy and environmental tobacco smoke on asthma and wheezing in children. Am J Respir Crit Care Med 2001, 163(2):429-36.

21. Campo-Arias. The prevalence of nicotine-dependency in some populations: a systematic review. Rev Salud Publica (Bogota). 2006;8(1):98-107. 